\title{
Photon blockade in a photonic crystal cavity with a strongly coupled quantum dot
}

\author{
Andrei Faraon*1, Ilya Fushman*, Dirk Englund*, Jelena Vučković \\ E.L. Ginzton Laboratory, Stanford University, Stanford, CA, 94305, USA \\ *. These authors contributed equally to this work
}

\begin{abstract}
The strong coupling regime between a single emitter and the mode of an optical resonator allows for nonlinear optics phenomena at extremely low light intensities. Down to the single photon level, extreme nonlinearities can be observed, where the presence of a single photon inside the resonator either blocks or enhances the probability of subsequent photons entering the resonator. In this paper we experimentally show the existence of these phenomena, named photon blockade and photon induced tunneling, in a solid state system composed of a photonic crystal cavity with a strongly coupled quantum dot.
\end{abstract}

Keywords: quantum information, photonic crystal, quantum dot

\section{INTRODUCTION}

Photonic crystals and quantum dots represent a scalable platform for implementing nano-scale devices for classical and quantum information science. ${ }^{1,2}$ We have recently developed techniques for local tuning of quantum dots and photonic crystal resonators. These techniques have been used to tune single quantum dots into resonance with a photonic crystal cavity, and observed strong coupling both in photoluminescence ${ }^{3-5}$ and in resonant light scattering from the system, ${ }^{6}$ as needed for several proposals for scalable quantum information networks ${ }^{7-9}$ and quantum computation. This system allows for solid-state implementation of nonlinear optical devices at the single photon level. Here we present the first experimental proof of photon blockade ${ }^{10,11}$ and photon-induced tunneling in a solid state cavity QED system.

\section{PHOTON BLOCKADE AND PHOTON-INDUCED TUNNELING}

The optical system consists of a three hole defect ${ }^{12}$ photonic crystal cavity (quality factor $Q=10000$ ) coupled to a self-assembled InAs quantum dot (QD) with decay rate $\gamma / 2 \pi=0.1 \mathrm{GHz}$, as described in Ref.6. The QD-cavity coupling rate $g / 2 \pi=16 \mathrm{GHz}$ equals the cavity field decay rate $\kappa / 2 \pi=16 \mathrm{GHz}$, putting the system in the strong coupling regime. ${ }^{4,5}$ In Fig.1(b) we show the photoluminescence scans where the anticrossing characteristic of strong coupling between the QD and the cavity can be observed. Here, the QD is tuned into resonance using local temperature tuning ${ }^{3}$ around an average temperature of $20 \mathrm{~K}$ maintained in a continuous He flow cryostat.

In order to observe the optical nonlinearities, coherent probing of the cavity - quantum dot system is required. The coherent probing was done using linearly polarized beams and the output was observed in cross-polarization as shown in Fig.1(a) and described in Ref.6. The cross-polarized setup allows us to separate the cavity coupled signal from the direct probe reflection, which is essential for achieving large signal to noise ratios needed in autocorrelation measurements. Our setup is such that the measurement on the reflected port from this singlesided cavity is analogous to a transmission measurement in a Fabry Perot arrangement.

The energy eigenstates of a two-level system strongly coupled on resonance to an optical resonator are grouped into two-level manifolds denoted $| \pm, n\rangle$, with energies $\hbar \omega_{n, \pm}=\hbar\left(n \omega_{0} \pm g \sqrt{n}\right)$, where $n$ is the number of energy quanta in the system and $\omega_{0}$ is the bare cavity frequency (Fig.2(b)). In Fig.2(a) we show schematically the energy diagram for the ground state and the first and second order manifold of the excited state. The anharmonic energy level spacing causes phenomena such as photon blockade ${ }^{10}$ or photon-induced tunneling. To observe photon blockade, a coherent probe beam (frequency $\omega_{p}$ ) tuned to $\omega_{1, \pm}=\omega_{0} \pm g$ is coupled to the cavity (blue arrow in Fig.2(a)). This probe is resonant with the first-order manifold, but detuned from transitions to

${ }^{1}$ To whom correspondence should be addressed. Email: faraon@stanford.edu 


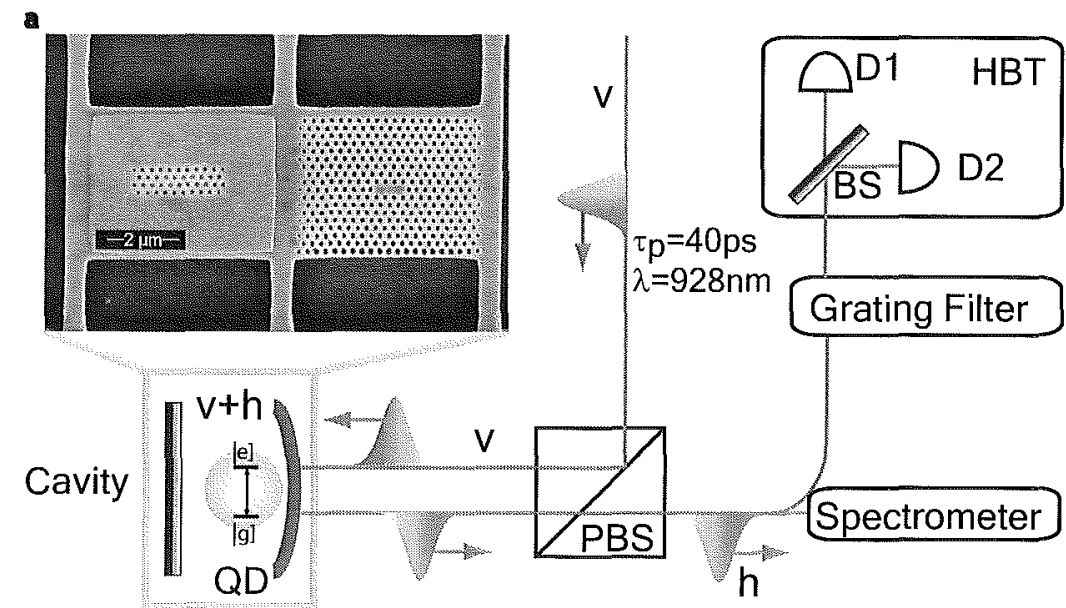

$\mathrm{b}$

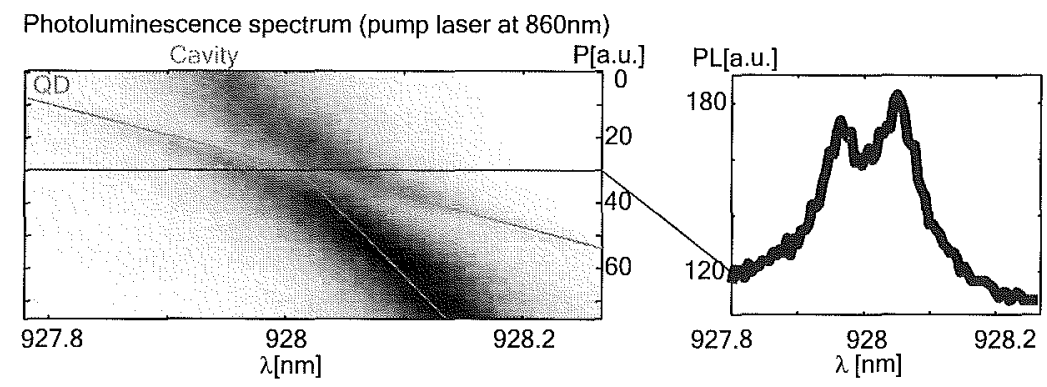

Figure 1. Schematic diagram of the experimental setup. (a) Laser pulses (40ps FWHM) are reflected from a GaAs photonic crystal cavity $(\mathrm{Q}=10000, \kappa / 2 \pi=16 \mathrm{GHz})$ strongly coupled to a InAs quantum $\operatorname{dot}(g / 2 \pi=16 \mathrm{GHz}, \gamma / 2 \pi=0.1 \mathrm{GHz})$. The cavity is linearly polarized at $45^{\circ}$ relative to the input polarization set by the polarizing beam splitter (PBS). The output light, observed in cross-polarization and carrying the cavity-coupled signal, is analyzed using a Hanbury-Brown-Twiss (HBT) setup that measures second-order correlation. The inset shows the suspended structure with the photonic crystal cavity and the metal pad for local temperature tuning.(b) Anticrossing observed in photoluminescence as the QD is tuned into resonance with the cavity. The temperature tuning is done by linearly increasing the power $(\mathrm{P})$ of a heating laser focused on the heating pad. The right inset shows the spectrum at the anticrossing point marked by the blue line. The red lines mark the cavity and QD resonance as if they were decoupled. 


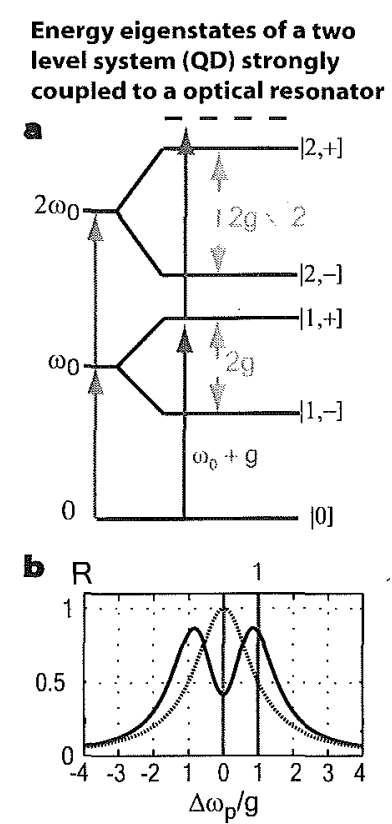

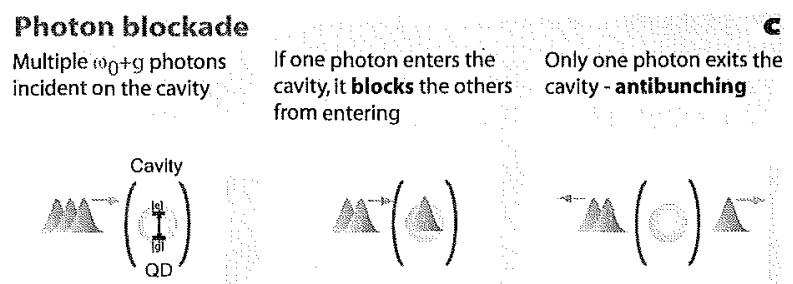

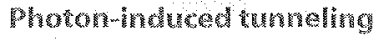

Multiple $\%$ photons If one photon enters the cavity, Photons exit the cavity

incident on the cavity it enhances the probability of other in bunches photons to tunnel in the cavity.

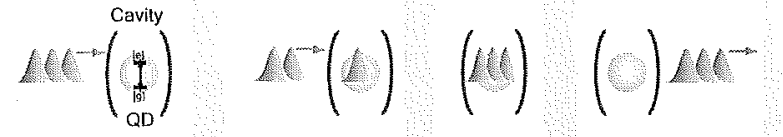

Shematic representation of photon blockade and photoninduced tunneling in a trasmission configuration (similar to a cross-polarized reflectivity measurement.). The signatures of photon blockade and photon-induced tunneling are antibunching and bunching respectively. These can be measured in a HBT photon auto-correlation measurement.

Figure 2. (a) The energy eigenstates of a two-level system strongly coupled on resonance to an optical resonator are grouped into two-level manifolds denoted $|n,+-\rangle$, with energies $\hbar\left(n \omega_{0} \pm g, n\right)$, where $\mathrm{n}$ is the number of energy quanta in the system, w0 is the bare cavity frequency and $g$ is the coupling strength. The anharmonic energy level spacing causes phenomena such as photon blockade and photon-induced tunneling. (b) Transmission spectrum (continuous line) of a cavity strongly coupled to a quantum dot. The dashed line shows the bare cavity transmission. $\Delta \omega_{p}$ is the detuning between the probe beam and the cavity resonance $\omega_{0}$.(c) Schematic explanation of photon blockade and photon-induced tunneling

the second manifold, $\omega_{1 \rightarrow 2}=\omega_{0} \pm g(\sqrt{2}-1)$ as shown in Fig.2(a). Consequently, once a photon is coupled into the system, it suppresses the probability of coupling a second photon with the same frequency. As a result, the output field acquires sub-poissonian statistics. In addition to photon blockade, photon-induced tunneling is expected near the bare cavity resonance $\left(\omega_{p}-\omega_{0}=\Delta \omega_{p} \rightarrow 0\right)$. An intuitive explanation of the tunneling effect is given in Fig.2(c). By tuning the resonance frequency closer to the bare cavity resonance, the probe beam becomes resonant with an $n$ photon transition to the $n^{\text {th }}$ order manifold. This way the probability of coupling the first photon into the system is lower than in the blockade case. However, once this first photon is coupled, the probability of coupling the second photon becomes enhanced because the probe is closer to the resonant transition between the first and second manifold. The probability of coupling into the next level keeps increasing until the $n^{\text {th }}$ order manifold is reached. This way, it is preferential to couple more photons into the system, so the output consists of "photon bunches." These phenomena are purely quantum effects that can not be explained using semi-classical theories. To probe them, one needs to measure the second order correlation function, $g^{(2)}(\tau)$. The signature of the photon blockade effect is the antibunching in $g^{(2)}(\tau)$ (i.e. $g^{(2)}(0)$ is a local minimum, $g^{(2)}(0)<g^{(2)}(\tau)$ ), as recently demonstrated by Birnbaumm et al ${ }^{10}$ in an experiment with neutral atoms. In the case of photon induced tunneling $g^{(2)}(0)$ is a local maximum.

During the experiment we scan several cavities until we find one which contains a strongly coupled QD, as determined by the anticrossing behavior in photoluminescence between QD and cavity during temperature tuning. Then we direct the pulsed laser beam at the cavity and observe the reflected beam in cross-polarization. While tuning the local temperature with an additional heating beam, we adjust the probe beam coupling to optimize the QD-induced reflectivity drop, as described for the continuous-wave beam in ref.6. Then we stop scanning and temperature-tune the QD and cavity onto resonance. With the pulsed probe beam at different detunings with respect to the anticrossing point, we measure the autocorrelation signal by passing the reflected 
probe through a grating filter (to remove stray light) followed by the HBT setup. To limit sample drift, the alignment procedure is repeated for every data point in Fig. 4.

We measure the time-dependent autocorrelation $g^{(2)}(\tau)$ using the Hanbury-Brown-Twiss (HBT) setup shown in Fig.1(a) and described in ref.[13,14]. The relevant features occur at time scales that correspond to the QDcavity coupling rate $g$, enveloped by the coherence time. ${ }^{15}$ The coherence time for our system is given by the cavity photon lifetime $1 / 2 \kappa \sim 5$ ps. Hence, the time-dependent features in $g^{(2)}(\tau)$ occur much faster than the 300 ps time-resolution of the single photon counting modules in the HBT setup. In order to resolve the relevant features, we sample the autocorrelation function by short pulses $\left(\Delta t_{F W H M} \sim 40 \mathrm{ps}, \Delta \omega_{F W H M} / 2 \pi \sim 12 \mathrm{GHz}\right)$ with a repetition rate of $12.5 \mathrm{~ns}$. This probe pulse duration represents a compromise between fast sampling and a spectral linewidth narrow enough to resolve the relevant spectral features. In the remainder of the paper we present the measurements of $g^{(2)}(\tau)$ for different detunings of the probe beam, denoted as $g^{(2)}\left(\tau, \Delta \omega_{p} / g\right)$.

The experimental data sets showing photon blockade and photon-induced tunneling in our system are shown in Fig.3. In the blockade case, we tuned the laser at $\Delta \omega_{p} / g=1.5$. We did not choose $\Delta \omega_{p} / g=1$ because we theoretically estimated that for $\Delta \omega_{p} / g=1$ the blockade effect should be more pronounced (see. Fig.4(a)). In the case of photon-induced tunneling we used $\Delta \omega_{p} / g=0$. In case of photon blockade, photon antibunching is observed at zero time delay, while for photon-induced tunneling bunching is observed (Fig.3(b,d)). Beside the effect visible at zero time delay, the histograms also show bunching over timescales of hundreds of nanoseconds. This bunching is a purely classical effect that results from the Poissonian blinking of the QD. As reported by Santori et al, ${ }^{16}$ such blinking is caused by quantum dot transitions between a bright and a dark state, and results in bunching near $\tau=0$ that falls off with the mean switching rate. Our observations indicate that the blinking rates vary for different QDs. The QD measured in this experiment spends $\sim 80 \%$ of the time in the bright state.

In the case of photon blockade, the value of the normalized second order correlation function is $\bar{g}^{(2)}(0,1.5)=$ $0.912 \pm 0.005$, reflecting the subpoissonian nature of the output field. For photon-induced tunneling we measure $\bar{g}^{(2)}(0,0)=1.37 \pm 0.02$ (Fig.3(d)). The overline in the notation for the second order correlation function is used to emphasize that the quantity that we measure is not exactly the second order correlation function at zero time delay. This is caused by the finite duration and bandwidth of the pulses used to probe the system and the finite temporal resolution of the photodetectors. The data is normalized such that $\bar{g}^{(2)}\left(\tau \rightarrow \infty, \Delta \omega_{p} / g\right)=1$.

By changing the frequency of the probe laser, we were able to measure the full spectrum of the second order correlation function. The results of this measurement is shown in Fig.4(b). In panel (a) of the same figure we also plot the expected value for the second order correlation spectrum. This is calculated considering a continuous wave laser beam probing the system that injects an average photon number of 0.4 into the system. The two curves are qualitatively similar, but there are differences in the absolute value that we measure for $\bar{g}^{(2)}\left(0, \Delta \omega_{p} / g\right)$. There are several factors that account for the difference between the theoretically predicted (Fig.4(a)) and measured values for $\bar{g}^{(2)}\left(0, \Delta \omega_{p} / g\right)$ : background caused by the imperfect extinction of the crosspolarized experimental setup (signal to noise ratio $\sim 6: 1$ ), QD blinking and finite bandwidth of the probe that affects the spectral resolution. Both the background and the output signal when the QD is in the dark state result in a flat second order correlation with $\bar{g}^{(2)}\left(\tau, \Delta \omega_{p} / g\right)=1$ (coherent light). With the quantum dot in the dark state, the cavity reflectivity becomes that of an empty cavity. Thus, when the QD transitions from the bright to the dark state the output field experiences intensity fluctuations. These intensity fluctuations cause the classical bunching observed over time scales of hundreds of nanoseconds. We modeled the experimental system by taking into consideration the finite bandwidth of the pulses, QD blinking and background from the imperfect extinction of the cross-polarized setup. The simulation results are shown in Fig.4(b), in very good agreement with the experimental data.

The experimental data in Fig.4 shows that, starting from a coherent state, the strongly coupled system allows control of the statistics of the output field from sub-poissonian to super-poissonian. Thus, by engineering the parameters of the system and by choosing the appropriate probe frequency one could generate various nonclassical states of light on demand. One of the most useful states is the single photon state that has applications in quantum cryptography and distributed quantum networking.

Using the anharmonicity of the eigenenergy spacing in this system, a single photon transistor, ${ }^{17}$ could be implemented. For example, the frequency of the gate field is resonant with one of the polaritons in the first-order 

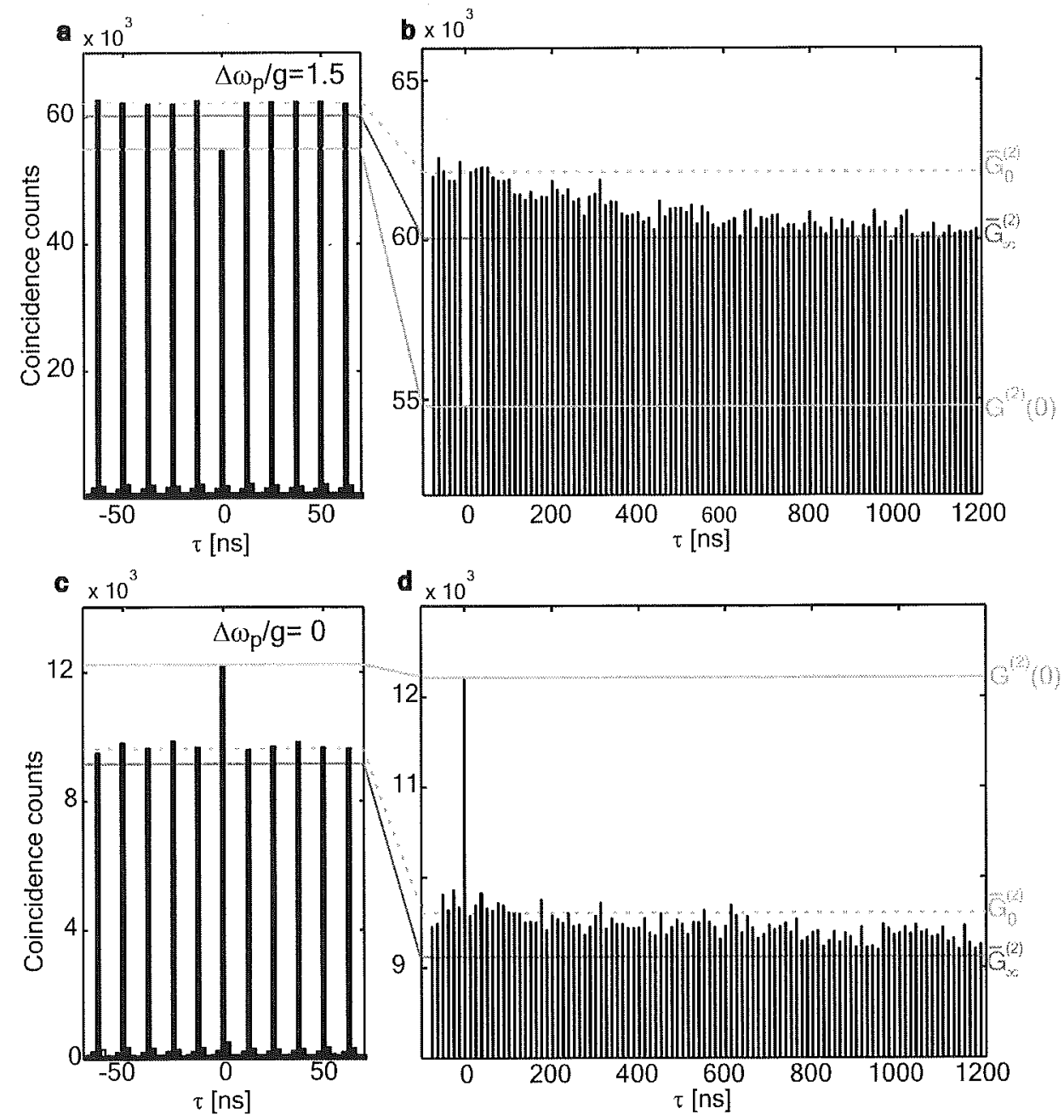

d $\times 10^{3}$

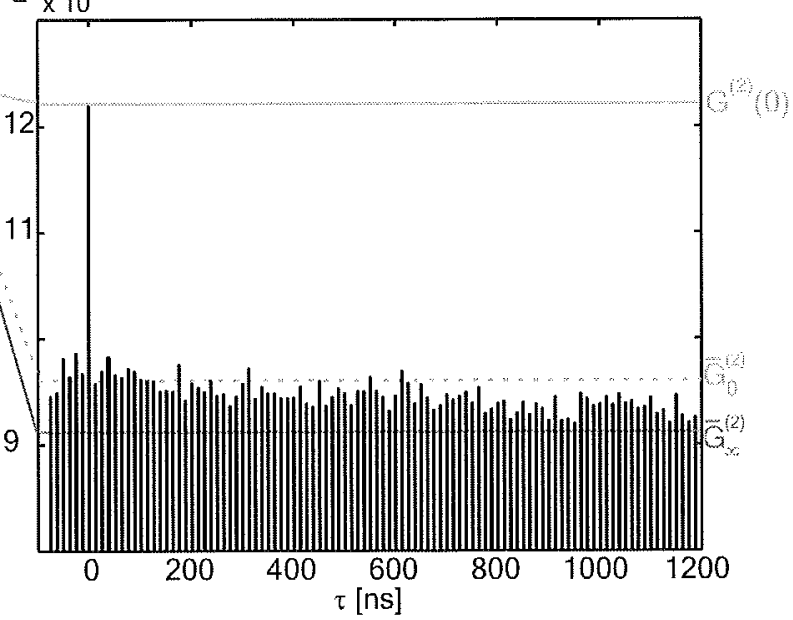

Figure 3. Measurement of the second-order correlation function for coherent laser pulses reflected from the photonic crystal cavity with a strongly coupled QD. (a,b) Photon blockade, manifested here in the sub-poissonian statistics with $\mathrm{g}(2) 0=0.91$. Complete antibunching could be observed for resonators with higher quality factors. (c,d) Photon-induced tunneling observed when the laser pulse is tuned to the anticrossing point. The measured non-classical bunching has $g(2) 0=1.37$. Remark: Beside the non-classical bunching and antibunching, bunching over longer timescales can be observed in (b) and (d). This bunching is caused by the classical blinking of the quantum dot between a dark and bright state. 

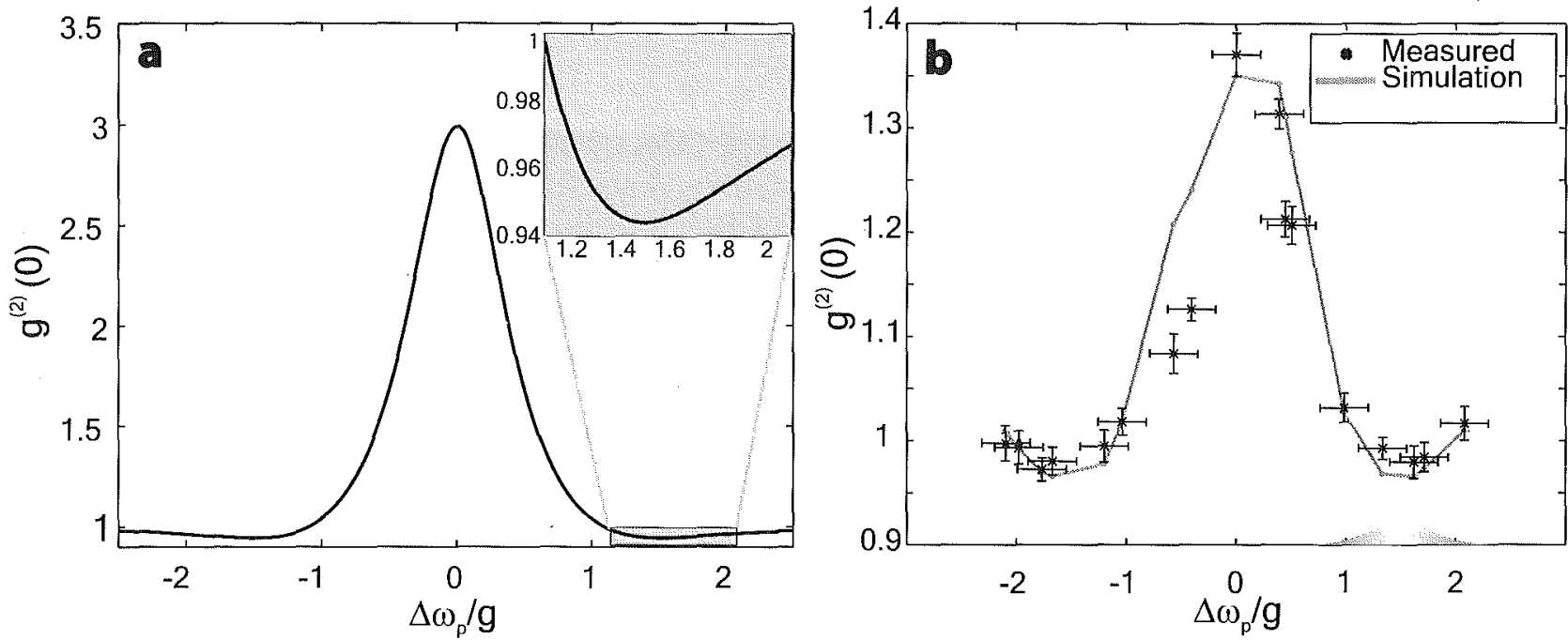

Figure 4. Second order correlation of the output field as the input probe frequency is scanned through the strongly coupled system. (a) Expected second order correlation when a weak continuous beam is used as a probe. The simulation assumes a cavity with $\kappa / 2 \pi=16 \mathrm{GHz}, g / 2 \pi=16 \mathrm{GHzandg} / 2 \pi=0.1 \mathrm{GHz}$. The inset shows the antibunching from photon blockade. The blockade is best observed at $\Delta \omega_{p} / g=1.5$ because for $\mathrm{Q}=10000$ there is significant overlap between consecutive manifolds of the eigenstates. Photon induced tunneling is the cause of bunching at zero detuning. (b) Measured second order correlation in pulsed regime. The red line shows the expected result taking into account pulsed operation and the sources of noise in the system.

manifold, say $\omega_{0}+g$. A photon injected at $\omega_{0}+g$, increases the probability of absorbing photons that are resonant with the $|1,+\rangle \rightarrow|2,+\rangle$ transition at $\omega_{0}+g(\sqrt{2}-1)$. If the signal is tuned to this frequency, the presence of the gate field enhances the transmission of the signal field. ${ }^{18}$ Photonic crystals are ideally suited for an integrated architecture, where cavities and waveguides are interconnected on the chip. One of the most straight forward implementations of the single photon transistor would be a photonic crystal resonator coupled to three photonic crystal waveguides.

\section{CONCLUSION}

In conclusion, we demonstrated photon blockade and photon induced tunneling in a photonic crystal cavity with a strongly coupled quantum dot. This opens the possibility of generating non-classical light on a chip using strongly coupled light-matter systems. These states are interesting for quantum information processing devices. The nonlinearities at single photon level can be used for implementing on-chip single photon transistors and for on-chip optical logic devices operating at very low power levels.

\section{REFERENCES}

1. Daniel Loss and David P. DiVincenzo. Quantum computation with quantum dots. Physical Review A, 57(1):120-126, 1998.

2. A. Imamoglu, D. D. Awschalom, G. Burkard, D. P. DiVincenzo, D. Loss, M. Sherwin, and A. Small. Quantum Information Processing Using Quantum Dot Spins and Cavity QED. Physical Review Letters, 83(20):4204-4207, November 1999.

3. Andrei Faraon, Dirk Englund, Ilya Fushman, Nick Stoltz, Pierre Petroff, and Jelena Vuckovic. Local quantum dot tuning on photonic crystal chips. Applied Physics Letters, 90(213110), 2007.

4. K. Hennessy, A. Badolato, M. Winger, D. Gerace, M. Atatüre, S. Gulde, S. Falt, E.L. Hu, and A. Imamoglu. Quantum nature of a strongly coupled single quantum dot-cavity system. Nature, 445:896-899, Feb 2007. 
5. T. Yoshie, A. Scherer, J. Hendrickson, G. Khitrova, H. M. Gibbs, G. Rupper, C. Ell, O. B. Shchekin, and D. G. Deppe. Vacuum Rabi splitting with a single quantum dot in a photonic crystal nanocavity. Nature, 432:200-203, November 2004.

6. Dirk Englund, Andrei Faraon, Ilya Fushman, Nick Stoltz, Pierre Petroff, and Jelena Vuckovic. Controlling cavity reflectivity with a single quantum dot. Nature, 450(7171), 2007.

7. J. I. Cirac, P. Zoller, H. J. Kimble, and H. Mabuchi. Quantum State Transfer and Entanglement Distribution among Distant Nodes in a Quantum Network. Physical Review Letters, 78(16):3221-24, April 1997.

8. D. Felinto, C. W. Chou, J. Laurant, E. W. Schomburg, H. De Riedmatten, and H. J. Kimble. Conditional control of the quantum states of remote atomic memories for quantum networking. Nature Physics, 2:844$848,2006$.

9. D. L. Moehring, P. Maunz, S. Olmschenk, K. C. Younge, D. N. Matsukevich, L.-M. Duan, and C. Monroe. Entanglement of single-atomquantumbits at adistance. Nature, 449:68-72, 2007.

10. K. M. Birnbaum, A. Boca, R. Miller, A. D. Boozer, T. E. Northup, and H. J. Kimble. Photon blockade in an optical cavity with one trapped atom. Nature, 436:87-90, 2005.

11. A. Imamoglu, H. Schmidt, G. Woods, and M. Deutsch. Strongly Interacting Photons in a Nonlinear Cavity. Physical Review Letters, 79(8):1467-1470, August 1997.

12. Y. Akahane, T. Asano, B.-S. Song, and S. Noda. High-Q photonic nanocavity in a two-dimensional photonic crystal . Nature, 425(6961):944-947, October 2003.

13. D. Englund, A. Faraon, B. Zhang, Y. Yamamoto, and J. Vučković. Generation and Transfer of Single Photons on a Photonic Crystal Chip. Optics Express, 15(9), April 2007.

14. D. Englund, D. Fattal, E. Waks, G. Solomon, B. Zhang, T. Nakaoka, Y. Arakawa, Y. Yamamoto, and J. Vučković. Controlling the Spontaneous Emission Rate of Single Quantum Dots in a Two-Dimensional Photonic Crystal. Physical Review Letters, 95(013904), July 2005.

15. H.J. Carmichael, R. J. Brecha, and P. R. Rice. Quantum interference and collapese of the wavefunction in cavity QED. Optics Communications, 82(1,2):73-79, April 1991.

16. Charles Santori, David Fattal, Jelena Vuckovic, Glenn S. Solomon, Edo Waks, and Yoshihisa Yamamoto. Submicrosecond correlations in photoluminescence from InAs quantum dots. Physical Review B, 69(205324), 2004.

17. Darrick E. Chang, Anders S. Sorense, Eugene A. Demler, and Mikhail D. Lukin. A single-photon transistor using nanoscale surface plasmons. Nature Physics, 3:807-812, November 2007.

18. R. J. Thompson, Q. A. Turchette, O. Carnal, and H. J. Kimble. Nonlinear spectroscopy in the strongcoupling regime of cavity QED. Phys. Rev. A, 57(4):3084, 1998. 